\title{
BOUNDEDNESS IN LINEAR TOPOLOGICAL SPACES
}

\author{
BY \\ S. SIMONS
}

Introduction. Throughout this paper we use the symbol $X$ for a (real or complex) linear space, and the symbol $F$ to represent the basic field in question. We write $R^{+}$for the set of positive (i.e., $\geqq 0$ ) real numbers. We use the term linear topological space with its usual meaning (not necessarily $T_{1}$ ), but we exclude the case where the space has the indiscrete topology (see $[1,3.3, \mathrm{pp}$. 123-127]). A linear topological space is said to be a locally bounded space if there is a bounded neighbourhood of 0 -which comes to the same thing as saying that there is a neighbourhood $U$ of 0 such that the sets $\{(1 / n) U\}(n=1,2, \cdots)$ form a base at 0 .

In $\$ 1$ we give a necessary and sufficient condition, in terms of invariant pseudometrics, for a linear topological space to be locally bounded. In $\$ 2$ we discuss the relationship of our results with other results known on the subject. In $\$ 3$ we introduce two ways of classifying the locally bounded spaces into types in such a way that each type contains exactly one of the $l^{p}$ spaces $(0<p \leqq 1)$, and show that these two methods of classification turn out to be identical. Also in $\$ 3$ we prove a metrization theorem for locally bounded spaces, which is related to the normal metrization theorem for uniform spaces, but which uses a different induction procedure. In $\S 4$ we introduce a large class of linear topological spaces which includes the locally convex spaces and the locally bounded spaces, and for which one of the more important results on boundedness in locally convex spaces is valid.

One final word on terminology: if $f: X \rightarrow R^{+}$is a function and $f(0)=0$ we shall say that $f$ gives the topology $u$ if, for each $x \in X$, the sets $\{y: y \in X, f(y-x)<1 / n\}(n=1,2, \cdots)$ form a base for $u$ at $x$. In general $f$ is not continuous with respect to the topology it gives. We shall say that the functions $f: X \rightarrow R^{+}$and $g: X \rightarrow R^{+}$are equivalent if they give the same topology, i.e., the filter bases $\{x: x \in X, f(x)<1 / n\} \quad(n=1,2, \cdots)$ and $\{x: x \in X, g(x)<1 / n\}$ $(n=1,2, \cdots)$ are equivalent. If $(X, u)$ is a linear topological space and $f: X \rightarrow R^{+}$ is a function such that the filter base $\{x: x \in X, f(x)<1 / n\} \quad(n=1,2, \cdots)$ is equivalent to the filter of $u$-neighbourhoods of 0 then $f$, in fact, gives the topology $u$.

1. Locally bounded spaces and $k$-pseudometrics.

Definition 1. We shall say that the function $f: X \rightarrow R^{+}$is an invariant pseudometric if

Received by the editors May 20, 1963. 
there exists $x \in X$ such that $f(x) \neq 0$,

$$
\begin{aligned}
& f(0)=0, f(-x)=f(x) \text { for all } x \in X, \text { and } \\
& f(x+y) \leqq f(x)+f(y) \text { for all } x, y \in X .
\end{aligned}
$$

(Strictly speaking, this involves a slight abuse of the usual meaning of the word "pseudometric," as $\rho(x, y)=f(x-y)$ is actually the pseudometric.)

We shall say that the function $f: X \rightarrow R^{+}$is a $k$-pseudometric, where $0<k \leqq 1$, if $f$ is an invariant pseudometric and (1) is replaced by the stronger postulate

$$
f(\lambda x)=|\lambda|^{k} f(x) \text { for all } \lambda \in F \text { and } x \in X .
$$

If $f$ is an invariant pseudometric on $X$ then the topology given on $X$ by $f$ makes $X$ into a topological group, and $f$ is continuous with respect to it.

If $f$ is a $k$-pseudometric on $X$ then the topology given on $X$ by $f$ makes $X$ into a locally bounded linear topological space-the set $\{x: x \in X, f(x)<1\}$ is a bounded, open neighbourhood of 0 .

A 1-pseudometric is the same thing as a seminorm.

LEMMA 1. We suppose that $U$ is a balanced absorbing proper subset of $X$ and $\mu \in R^{+}$is such that $U+U+U \subset \mu U(\mu \geqq 3)$. We write $k$ for $\log _{\mu} 2$.

(a) For $x \in X$ we define $d(x)=\inf \left\{\lambda^{k}: \lambda \in R^{+}, x \in \lambda U\right\}$. Then, for $x, y, z \in X$ and $\lambda \in R^{+}$,

$$
d(x), d(y) \text { and } d(z) \leqq \lambda \text { imply that } d(x+y+z) \leqq 2 \lambda .
$$

(b) We suppose that $d$ is as above and, for $x \in X$, we define $f(x)$ to be $\inf \left\{\sum d\left(x_{i}\right)\right\}$, with $\left\{x_{i}\right\}$ ranging over all finite subsets of $X$ such that $\sum x_{i}=x$. Then $f$ is a k-pseudometric and, for each $x \in X$,

$$
\frac{1}{2} d(x) \leqq f(x) \leqq d(x)
$$

Proof. The only statement that really requires any proof is the inequality $\frac{1}{2} d(x) \leqq f(x)$ in (b). It clearly suffices to show that $d\left(\sum x_{i}\right) \leqq 2 \sum d\left(x_{i}\right)$ for all finite subsets $\left\{x_{i}\right\}$ of $X$. This can be done by induction on the number of elements in $\left\{x_{i}\right\}$, using (a), in a fashion similar to that employed in the normal metrization theorem for uniform spaces. (See [2, §1, No. 4, Proposition 2, p. 8]).

THEOREM 1. For a linear topological space, $X$, to be locally bounded it is necessary and sufficient that the topology of $X$ be given by a $k$-pseudometric for some $k, 0<k \leqq 1$.

Proof. The "sufficiency" has already been commented on in the remarks following Definition 1 . If, conversely, $V$ is a bounded neighbourhcod of 0 in $X$ then we can choose a balanced neighbourhood $U$ of 0 such that $U \subset V$. Clearly $U$ is bounded and so, from the continuity of $x+y+z$ at $(0,0,0)$, there exists 
a strictly positive integer $n$ such that $(1 / n) U+(1 / n) U+(1 / n) U \subset U$. Since the topology of $X$ is not indiscrete, $U \neq X$. We define the function $f: X \rightarrow R^{+}$as in Lemma 1 , with $\mu=n$. Then $f$ is a $k$-pseudometric and $\left\{x: x \in X, f(x)<\frac{1}{2}\right\}$ $\subset U \subset\{x: x \in X, f(x) \leqq 1\}$. The result follows.

REMARK. The result of Theorem 1 answers some questions raised in Chapter III of [5]. We will prove a stronger result in $\$ 3$ (Theorem 2).

LEMMA 2. If $f$ is a k-pseudometric on $X$ and $0<m \leqq 1$ then $f^{m}$ is an mk-pseudometric on $X$.

Proof. For $t \geqq 0$ the function $t \rightarrow t^{m}$ is increasing and subadditive. Thus, for $x, y \in X$,

$$
f^{m}(x+y) \leqq[f(x)+f(y)]^{m} \leqq f^{m}(x)+f^{m}(y) .
$$

The other postulates for $f^{m}$ to be an $m k$-pseudometric are trivially satisfied.

COROLlary. If $f$ is a $k$-pseudometric on $X$ and $0<k^{\prime} \leqq k$ then there is a $k^{\prime}$-pseudometric on $X$ equivalent to $f$.

Proof. It follows from the lemma that if $m$ is chosen so that $m k=k^{\prime}$ then $f^{m}$ is a $k^{\prime}$-pseudometric. It is immediate that $f^{m}$ is equivalent to $f$.

The $l^{p}$ spaces. We suppose that $0<p<1$ and write $l^{p}$ for the set of real (or complex) sequences $\left(x_{v}\right)$ such that $\sum\left|x_{v}\right|^{p}<\infty$. The "usual" topology, $u^{p}$, of $l^{p}$ is defined by the $p$-pseudometric

$$
\left(x_{v}\right) \rightarrow \sum\left|x_{v}\right|^{p}
$$

$\left(l^{p}, u^{p}\right)$ is a complete, $T_{1}$, locally bounded space and is nct locally convex.

For each integer $n \geqq 1$ we write $e_{n}$ for the element

$$
(0,0, \cdots, 0,1,0, \cdots)
$$

of $l^{p}$, where the unit element comes in the $n$th place, and $h_{n}$ for

$$
n^{-1 / p} \sum_{v=1}^{n} e_{v}
$$

LEMmA 3. (a) If $0<k \leqq p$ then there exists a $k$-pseudometric on $l^{p}$ giving the topology $u^{p}$.

(b) If $f$ is a $k$-pseudometric on $l^{p}$ giving a topology as coarse as $u^{p}$ then the sequence $\left\{f\left(e_{n}\right)\right\}$ is bounded.

(c) If $k>p$ and $f$ is a $k$-pseudometric on $l^{p}$ giving a topology as fine as $u^{p}$ then the sequence $\left\{f\left(e_{n}\right)\right\}$ is unbounded.

(d) If $k>p$ then there is no $k$-pseudometric on $l^{p}$ giving the topology $u^{p}$.

Proofs. (a) follows from the Corollary of Lemma 2.

To prove (b) we observe that there exists $\delta>0$ such that $\Sigma\left|x_{v}\right|^{p} \leqq \delta$ implies that $f\left(\left(x_{v}\right)\right) \leqq 1$. Thus, for each integer $n \geqq 1, f\left(\delta^{1 / p} e_{n}\right) \leqq 1$ and so $f\left(e_{n}\right) \leqq \delta^{-k / p}$. 
To prove (c) we observe that $h_{n} \nrightarrow 0$ in $u^{p}$ and so $f\left(h_{n}\right) \nrightarrow 0$ (as $\left.n \rightarrow \infty\right)$. Since $f$ is a $k$-pseudometric,

and so

$$
f\left(h_{n}\right) \leqq n^{-k / p} \sum_{v=1}^{n} f\left(e_{v}\right)
$$

$$
n^{-k / p} \sum_{v=1}^{n} f\left(e_{v}\right) \nrightarrow 0 \text { as } n \rightarrow \infty .
$$

It follows that the sequence $\left\{f\left(e_{n}\right)\right\}$ is unbounded.

(d) is immediate from (b) and (c).

2. Locally bounded spaces and quasi seminorms.

Definition 2. We shall say that the function $g: X \rightarrow R^{+}$is a quasi seminorm if

$$
\begin{aligned}
& \text { there exists } x \in X \text { such that } g(x) \neq 0, \\
& g(\lambda x)=|\lambda| g(x) \text { for all } \lambda \in F \text { and } x \in X
\end{aligned}
$$

and there exists $b \in R^{+}$such that

$$
g(x+y) \leqq b[g(x)+g(y)] \text { for all } x, y \in X .
$$

We refer to the smallest $b \in R^{+}$for which (2) is satisfied as the multiplier of $g$. Clearly $b \geqq 1$. A seminorm is the same thing as a quasi seminorm with multiplier 1 . (The term quasi seminorm is the natural generalization of the term quasi norm of $[3, \S 6$, p. 651].)

If $g$ is a quasi seminorm on $X$ then $g$ gives a topology on $X$ with respect to which $X$ is a locally bounded space. We can adapt the argument of [3, Theorem 13$, p. 651$]$ to the non- $T_{1}$ case and show that the topology of any locally bounded space can be given by a quasi seminorm. We leave the details to the reader.

Thus: a linear topological space is locally bounded if, and only if, there is a quasi seminorm giving its topology.

On the surface, this result is similar to that of Theorem 1. The difference lies in the fact that a quasi seminorm can be wildly discontinuous with respect to the topology it gives, contrasting strongly with the situation for invariant pseudometrics outlined in the remarks following Definition 1. For this reason, it is more convenient to have a condition in terms of invariant pseudometrics than quasi seminorms. For instance, the extension by closure of a $k$-pseudometric is a $k$-pseudometric, and so it follows directly from $[2, \S 2$, No. 1 , Proposition 1 , pp. 18-19] that the completion of a $T_{1}$ locally bounded space is again locally bounded. (Cf. [3, Theorem 15, pp. 652-653].)

The following connection between $k$-pseudometrics and quasi seminorms is interesting.

LEMMA 4. If $f: X \rightarrow R^{+}$is a $k$-pseudometric then $f^{1 / k}$ is a quasi seminorm with multiplier $\leqq 2^{(1 / k)-1}$. 
Proof. If $x, y \in X$ then $f(x+y) \leqq f(x)+f(y) \leqq 2^{1-k}\left[f^{1 / k}(x)+f^{1 / k}(y)\right]^{k}$, from Hölder's inequality. Thus

$$
f^{1 / k}(x+y) \leqq 2^{(1 / k)-1}\left[f^{1 / k}(x)+f^{1 / k}(y)\right] .
$$

The other postulates for $f^{1 / k}$ to be a quasi seminorm are trivially satisfied.

COROLlary. If $(X, u)$ is a locally bounded space then there is a continuous quasi seminorm on $(X, u)$ giving the topology $u$.

Proof. From Theorem 1, there exists a $k$-pseudometric $f$ on $X$ giving the topology $u$. Since it is a $k$-pseudometric, $f$ is continuous with respect to $u$. Then $f^{1 / k}$ is a quasi seminorm, gives the topology $u$ and is continuous with respect to $u$. (Cf. [3, Theorem 17, p. 653].)

LemMA 5. If $g$ is a quasi seminorm on $X$ with multiplier $b$ and $k=\log _{2 b} 2$ then, for every subset $\left\{x_{1}, \cdots, x_{n}\right\}$ of $n$ elements of $X$,

$$
g\left(x_{1}+\cdots+x_{n}\right) \leqq(2 n)^{(1 / k)-1}\left[g\left(x_{1}\right)+\cdots+g\left(x_{n}\right)\right]
$$

Proof. If $x_{1}, x_{2}, \cdots$ are elements of $X$ then

$$
\begin{aligned}
g\left(x_{1}+x_{2}\right) & \leqq b\left[g\left(x_{1}\right)+g\left(x_{2}\right)\right] \\
g\left(x_{1}+x_{2}+x_{3}+x_{4}\right) & \leqq b\left[g\left(x_{1}+x_{2}\right)+g\left(x_{3}+x_{4}\right)\right] \\
& \leqq b^{2}\left[g\left(x_{1}\right)+g\left(x_{2}\right)+g\left(x_{3}\right)+g\left(x_{4}\right)\right]
\end{aligned}
$$

and this argument can clearly be extended to show that, for any integer $n$ such that $1 \leqq n \leqq 2^{r}$,

$$
g\left(x_{1}+\cdots+x_{n}\right) \leqq b^{r}\left[g\left(x_{1}\right)+\cdots+g\left(x_{n}\right)\right] .
$$

If $n$ is an integer $>1$ we choose $r$ so that $n \leqq 2^{r}<2 n$. Then

$$
b^{r}=2^{[(1 / k)-1] r}=2^{r[(1 / k)-1]} \leqq(2 n)^{(1 / k)-1},
$$

since the choice of $k$ implies that $b=2^{(1 / k)-1}$. The result follows.

More properties of the $l^{p}$ spaces. We refer the reader to the definitions and notation introduced immediately before Lemma 3 . The following results for quasi seminorms on $l^{p}$ parallel those for $k$-pseudometrics proved in Lemma 3.

LeMma 6. (a) There is a quasi seminorm on $l^{p}$ with multiplier $2^{(1 / p)-1}$ giving the topology $u^{p}$.

(b) If $b<2^{(1 / p)-1}$ then there is no quasi seminorm on $l^{p}$ with multiplier $b$ giving the topology $u^{p}$.

Proof. The function $N\left(\left(x_{v}\right)\right) \equiv\left[\Sigma\left|x_{v}\right|^{p}\right]^{1 / p}$ is, from Lemma 4, a quasi seminorm on $l^{p}$ with multiplier $\leqq 2^{(1 / p)-1}$. On the other hand, $N\left(e_{1}\right)=1, N\left(e_{2}\right)=1$ and $N\left(e_{1}+e_{2}\right)=2^{1 / p}$; hence the multiplier of $N$ is exactly $2^{(1 / p)-1}$. This proves the truth of (a). 
If $g$ is a quasi seminorm on $l^{p}$ giving a topology as coarse as $u^{p}$ then, arguing as in Lemma $3(\mathrm{~b})$, the sequence $\left\{g\left(e_{n}\right)\right\}$ is bounded. If $g$ is a quasi seminorm on $l^{p}$ with multiplier $b, b<2^{(1 / p)-1}, k=\log _{2 b} 2$ (so that $k>p$ ) and $g$ gives a topology as fine as $u^{p}$ then, arguing as in Lemma 3(c) and using Lemma 5,

i.e.,

$$
(2 n)^{(1 / k)-1} n^{-1 / p} \sum_{v=1}^{n} g\left(e_{v}\right) \nrightarrow 0 \text { as } n \rightarrow \infty,
$$

$$
2^{(1 / k)-1} n^{(1 / k)-(1 / p)}\left[n^{-1} \sum_{v-1}^{n} g\left(e_{v}\right)\right] \nrightarrow 0 \text { as } n \rightarrow \infty,
$$

and thus the sequence $\left\{g\left(e_{n}\right)\right\}$ is unbounded. This proves the truth of (b).

\section{The classification of locally bounded spaces and a metrization theorem.}

Definition 3. If $(X, u)$ is a locally bounded space then we shall describe the number $p$,

$$
p=\sup \{k \text { :there exists a } k \text {-pseudometric on } X \text { giving the topology } u\},
$$
as the type of $(X, u)$. Clearly $0<p \leqq 1$.

It follows from Lemma 3 that $\left(l^{p}, u^{p}\right)$ is of type $p$. A seminormed space is of type 1.

What we have called a " $k$-pseudometric" appears in $[4$, p. 106] as a " $k$-seminorm," though we exclude the zero functional. It follows from (loc. cit.), Note 2, that if $(X, u)$ is a locally bounded space then the type of $(X, u)$ is equal to

$$
\sup \{k:(X, u) \text { is } k \text {-convex }\} \text {. }
$$

Using a space similar to $l\left(F,\left(p_{n}\right)\right)$ (op. cit., pp. 109-110) we can easily construct a locally bounded space which is of type 1 but not locally convex.

Definition 4. If $(X, u)$ is a locally bounded space we shall say that $b \in R^{+}$ is a multiplier for $(X, u)$ when there is a quasi seminorm on $X$ with multiplier $b$ giving the topology $u$. We shall write

$$
\beta(X, u)=\inf \{b: b \text { is a multiplier for }(X, u)\} .
$$

Clearly $\beta(X, u) \geqq 1$.

It follows from Lemma 6 that $\beta\left(l^{p}, u^{p}\right)=2^{(1 / p)-1}$.

We can regard both the type and also the number $\beta$ as a measurement of the extent by which a locally bounded space departs from being seminormed. Consequently, we get two natural methods of classifying locally bounded spaces. It is gratifying to know that these two methods turn out to be identical. Our purpose in this section is to prove this fact. The crucial point in the argument is Theorem 2. We state this now, but defer the proof for the moment.

THEOREM 2. If $g$ is a quasi seminorm on $X, g$ has multiplier $b$ and $m<\log _{2 b} 2$ then there exists an m-pseudometric on $X$ equivalent to $g$. 
THEOREM 3. (a) If $(X, u)$ is a locally bounded space of type $p$ then

$$
\beta=\beta(X, u)=2^{(1 / p)-1} .
$$

(b) The classifications of locally bounded spaces by type and by $\beta$ are equivalent.

Proof. If $f$ is a $k$-pseudometric on $X$ giving the topology $u$ then, from Lemma $4, \beta \leqq 2^{(1 / k)-1}$. Since we can take $k$ here as near as we please to $p$, it follows that $\beta \leqq 2^{(1 / p)-1}$.

If $c>\beta$ then there exists a quasi seminorm on $X$ with multiplier $b, b<c$, giving the topology $u . b<c$ implies that

$$
\log _{2 c} 2<\log _{2 b} 2
$$

and thus, from Theorem $2, \log _{2 c} 2 \leqq p$. Since we can take $c$ here as near as we please to $\beta, \log _{2 \beta} 2 \leqq p$, i.e., $\beta \geqq 2^{(1 / p)-1}$.

Thus $\beta=2^{(1 / p)-1}$, completing the proof of (a).

The maps $p \rightarrow 2^{(1 / p)-1}$ and $\beta \rightarrow \log _{2 \beta} 2$ define a $(1,1)$ correspondence between $p \in(0,1]$ and $\beta \in[1, \infty)$. Thus (b) follows from (a).

We now discuss the difficulties involved in the proof of Theorem 2. It is clear that, for $x, y, z \in X$,

$$
g(x+y+z) \leqq b^{2} g(x)+b^{2} g(y)+b g(z) .
$$

Consequently, provided $m$ is such that $\left(2 b^{2}+b\right)^{m} \leqq 2, g^{m}(x), g^{m}(y)$ and $g^{m}(x) \leqq \lambda$ imply that $g^{m}(x+y+z) \leqq 2 \lambda$ for all $x, y, z \in X$ and $\lambda \in R^{+}$. In this case, the argument mentioned in the proof of Lemma 1(b) can be used and yields that, for each $x \in X, f(x) \leqq g^{m}(x) \leqq 2 f(x)$, where

(3) $f(x)=\inf \left\{\sum g^{m}\left(x_{i}\right):\left\{x_{i}\right\}\right.$ ranging over all finite subsets of $X$ such that $\left.\sum x_{i}=x\right\}$.

This argument can be adapted slightly to prove that, provided $m$ is such that $\left(b^{2}+b\right)^{m} \leqq 2$, there exists a constant $M$ such that

$$
\text { for all } x \in X, f(x) \leqq g^{m}(x) \leqq M f(x),
$$

with $f(x)$ defined as in (3).

However, when $b$ is large there is quite a gap between the largest value of $m$ such that $\left(b^{2}+b\right)^{m} \leqq 2$ and $\log _{2 b} 2$, the bound for $m$ mentioned in Theorem 2 and needed for Theorem 3. It seems that too much information is lost by the split into three parts to allow us to deal with the cases when $m$ is near $\log _{2 b} 2$. The method to be presented here uses essentially a split into two parts and gives the desired result.

LemMA 7. We suppose that $g$ is a quasi seminorm on $X$ with multiplier $b$ and $m<\log _{2 b} 2$ (or, equivalently, $(2 b)^{m}<2$ ).

(a) There exists $\gamma, 0<\gamma<1$, such that 


$$
\gamma \leqq x \leqq 1 \text { implies that } 1+x-b^{m}\left(1+x^{1 / m}\right)^{m} \geqq 0 .
$$

(b) If $n$ is an integer $\geqq 1$ then there exists a constant $B_{n}$ such that

$$
g^{m}\left(x_{1}+\cdots+x_{n}\right) \leqq B_{n}\left[g^{m}\left(x_{1}\right)+\cdots+g^{m}\left(x_{n}\right)\right] \text { for all subsets }
$$$$
\left\{x_{1}, \cdots, x_{n}\right\} \text { of } n \text { elements of } X \text {. }
$$

(c) If $n$ is an integer $\geqq 1$ we write

$$
M_{n}=\inf \left\{B_{n}: B_{n} \text { satisfies (6) }\right\} \text {. }
$$

Then (6) in fact holds with $M_{n}$ in place of $B_{n}$. Furthermore,

where $\gamma$ is as in (a).

$$
M_{n+1} \leqq M_{n}\left(1+2 M_{2} \gamma^{n-1}\right) \text {, }
$$

(d) The sequence $\left\{M_{n}\right\}$ is bounded.

(e) There exists a constant $M$ such that

$$
g^{m}\left(x_{1}+\cdots+x_{n}\right) \leqq M\left[g^{m}\left(x_{1}\right)+\cdots+g^{m}\left(x_{n}\right)\right]
$$

for all finite subsets $\left\{x_{1}, \cdots, x_{n}\right\}$ of $X$.

Proofs. The function $t \rightarrow 1+t-b^{m}\left(1+t^{1 / m}\right)^{m}$ is continuous for $0 \leqq t \leqq 1$ and takes the value $2-(2 b)^{m}>0$ when $t=1$. Thus the result of (a) follows.

Lemma 5 and the subadditivity of the function $t \rightarrow t^{m}$ for $t \geqq 0$ give that

$$
g^{m}\left(x_{1}+\cdots+x_{n}\right) \leqq(2 n)^{(m / k)-m}\left[g^{m}\left(x_{1}\right)+\cdots+g^{m}\left(x_{n}\right)\right],
$$

where $k$ is as in Lemma 5. This establishes the truth of (b).

The truth of the first assertion of (c) is immediate. We now embark on the proof of the second assertion, which is the most difficult step in the lemma. We suppose that $\left\{x_{1}, \cdots, x_{n+1}\right\}$ is an arbitrary subset of $n+1$ elements of $X$. If necessary, we renumber them so that

If

$$
g^{m}\left(x_{1}\right) \leqq g^{m}\left(x_{2}\right) \leqq \cdots \leqq g^{m}\left(x_{n}\right) \leqq g^{m}\left(x_{n+1}\right) .
$$

$$
\text { there exists } i, 1 \leqq i \leqq n \text {, such that } \gamma g^{m}\left(x_{i+1}\right)<g^{m}\left(x_{i}\right)
$$

then $0<g^{m}\left(x_{i}\right)$, implying that $0<g^{m}\left(x_{i+1}\right)$ and so we can legitimately write

$$
\gamma \leqq \frac{g^{m}\left(x_{i}\right)}{g^{m}\left(x_{i+1}\right)} \leqq 1
$$

On applying (5) and clearing of fractions we get $b^{m}\left[g\left(x_{i}\right)+g\left(x_{i+1}\right)\right]^{m} \leqq g^{m}\left(x_{i}\right)$ $+g^{m}\left(x_{i+1}\right)$ and, since $b$ is the multiplier of $g$,

$$
g^{m}\left(x_{i}+x_{i+1}\right) \leqq g^{m}\left(x_{i}\right)+g^{m}\left(x_{i+1}\right) .
$$

Thus 


$$
g^{m}\left(\sum_{v=1}^{n+1} x_{v}\right) \leqq M_{n}\left[\sum_{v=1}^{i-1}+\sum_{v=i+2}^{n+1} g^{m}\left(x_{v}\right)+g^{m}\left(x_{i}+x_{i+1}\right)\right]
$$

and so, from (8),

$$
g^{m}\left(x_{1}+\cdots+x_{n+1}\right) \leqq M_{n}\left[g^{m}\left(x_{i}\right)+\cdots+g^{m}\left(x_{n+1}\right)\right] .
$$

If, on the other hand, (7) is not satisfied then,

$$
\text { for each } i, 1 \leqq i \leqq n, \gamma g^{m}\left(x_{i+1}\right) \geqq g^{m}\left(x_{i}\right)
$$

and so, in this case,

$$
g^{m}\left(x_{1}+x_{2}\right) \leqq M_{2}\left[g^{m}\left(x_{1}\right)+g^{m}\left(x_{2}\right)\right] \leqq 2 M_{2} \cdot \gamma^{n-1} g^{m}\left(x_{n+1}\right) .
$$

Here $g^{m}\left(x_{1}+\cdots+x_{n+1}\right) \leqq M_{n}\left[g^{m}\left(x_{1}+x_{2}\right)+\sum_{v=3}^{n+1} g^{m}\left(x_{v}\right)\right]$ and so, from (11),

$$
g^{m}\left(x_{1}+\cdots+x_{n+1}\right) \leqq M_{n}\left[1+2 M_{2} \gamma^{n-1}\right]\left[g^{m}\left(x_{1}\right)+\cdots+g^{m}\left(x_{n+1}\right)\right] .
$$

Given an arbitrary subset $\left\{x_{1}, \cdots, x_{n+1}\right\}$ of $n+1$ elements of $X$, either (7) or (10) must be satisfied and so either (9) or (12) must be satisfied. This clearly implies that $M_{n+1} \leqq\left(1+2 M_{2} \gamma^{n-1}\right) M_{n}$.

(d) is immediate from (c) and the fact that the product

is convergent.

$$
\prod_{n=1}^{\infty}\left(1+2 M_{2} \gamma^{n-1}\right)
$$

(e) follows from (d), where we take $M$ to be $\sup \left\{M_{n}\right\}$.

Proof of Theorem 2. For $x \in X$ we define $f(x)$ by (3). It follows from Lemma 7 (e) that (4) is satisfied, and this gives the desired result.

\section{Upper bound spaces.}

THEOREM 4. We suppose that $(X, u)$ is a linear topological space and that $0 \leqq q<1$. Then the conditions (a), (b) and (c) are equivalent.

(a) The topology $u$ is the upper bound of a family $\left\{u_{i}\right\}(i \in I)$ of locally bounded topologies of type $>q$ on $X$.

(b) There exists a family $\left\{f_{i}\right\} \quad(i \in I)$ of u-continuous $k_{i}$-pseudometrics $\left(q<k_{i} \leqq 1\right)$ on $X$ such that the sets $\left\{x: f_{i}(x)<1 / n\right\}(i \in I, n \in N)$ form a subbase : for $u$ at 0 . (We write $N$ for the set $\{1,2, \cdots\}$.)

(c) There exists a family $\left\{f_{j}\right\}(j \in J)$ of u-continuous $k_{j}$-pseudometrics $\left(q<k_{j} \leqq 1\right)$ on $X$ such that the sets $\left\{x: f_{j}(x)<1\right\}(j \in J)$ form a base for $u$ at 0 .

Proofs. Condition (a) means that there is a family $\left\{u_{i}\right\}(i \in I)$ of locally bounded topologies of type $>q$ on $X$ such that the sets $\left\{U_{i}\right\}$ form a subbase for $u$ at 0 , with $U_{i}$ running through all the $u_{i}$-neighbourhoods of 0 and $i$ running through $I$. It clearly suffices to take $U_{i}$ running through a basic set of $u_{i}$-neighbourhoods of 0 and thus, from Definition 3, (a) implies (b). (We observe thatis. 
since $u$ is finer than each $u_{i}$, any $u_{i}$-continuous $k$-pseudometric is also $u$-continuous.) It also follows without much difficulty that (c) implies (a). It remains to show that (b) implies (c).

If (b) is true we write $\mathscr{S}$ for the aggregate of all finite subsets of $I$ and $J$ for $N \times \mathscr{S}$. For $(n, S) \in J$ and $x \in X$ we write

where

$$
f_{(n, S)}(x)=n \max \left\{f_{i}^{k} S^{/ k_{t}}(x): i \in S\right\},
$$

$$
k_{s}=\min \left\{k_{s}: s \in S\right\} .
$$

It follows from Lemma 2 that $f_{(n, S)}$ is a $k_{s}$-pseudometric on $X$. Since $q<k_{i}$ for each $i \in I$ it follows that $q<k_{S}$ for each $S \in \mathscr{S}$. We leave it to the reader to show that $\left\{f_{(n, S)}\right\}((n, S) \in J)$ satisfy the conditions stated in (c).

Definition 5. We shall say that the linear topological space $(X, u)$ is an upper bound space if $u$ is the upper bound of a family of locally bounded topologies on $X$. In view of Theorem 4, with $q=0$, this is equivalent to the statement that there exists a family $\left\{f_{j}\right\}$ of $u$-continuous $k_{j}$-pseudometrics on $X$ such that the sets $\left\{x: f_{j}(x)<1\right\}$ form a base for $u$ at 0 . Clearly any locally convex or locally bounded space is an upper bound space.

THEOREM 5. If $(X, u)$ is a locally bounded space of type $p$ then the condition of Theorem 4 is satisfied for $q<p$ and not satisfied for $q \geqq p$.

Proof. The first observation is immediate from Definition 3. If $q \geqq p$ and condition (c) of Theorem 4 were satisfied then there would exist $k, p(\leqq q)<k \leqq 1$, and a $u$-continuous $k$-pseudometric $f$ on $X$ such that $\{x: f(x)<1\}$ was a bounded neighborhood of 0 in $u$. This would imply that $f$ gave the topology $u$, contradicting the original hypothesis that $(X, u)$ was of type $p$.

Definition 6. If $(X, u)$ is an upper bound space then we shall describe the number $p$,

$$
p=\sup \{q: q \text { satisfies the condition of Theorem } 4\}
$$

as the type of $(X, u)$. Clearly $0 \leqq p \leqq 1$.

A locally convex space is of type 1 and, from Theorem 5 , this definition of type is consistent with Definition 3 when $(X, u)$ is locally bounded. The linear topological space $(X, u)$ is $r$-convex (in the sense of [4]) if, and only if, there exists a family $\left\{f_{j}\right\}$ of $u$-continuous $r$-pseudometrics on $X$ such that the sets $\left\{x: f_{j}(x)<1\right\}$ form a base for $u$ at 0 . Using Lemma 2 we can deduce from this the following two results:

the type, $p$, of an upper bound space $(X, u)$ is given by the formula

$$
p=\sup \{r: 0<r<1,(X, u) \text { is } r \text {-convex }\},
$$

and 
the upper bound space $(X, u)$ is of type $\geqq p$ if, and only if, $(X, u)$ is $r$-convex for each $r$ such that $0<r<p$.

REMARK. All the results so far proved in this section (and some of those yet to be proved) for $k$-pseudometrics have analogues in terms of quasi seminorms. Theorem 3 can be used to pass between them. Details are left to the reader.

Subspaces, quotients and completions. Let $\mathscr{A}$ consist of any of the following classes of linear topological spaces:

the $r$-convex spaces, where $r$ is fixed, $0<r \leqq 1$,

the locally bounded spaces of type $\geqq p$, where $p$ is fixed, $0<p \leqq 1$, the upper bound spaces of type $\geqq p$, where $p$ is fixed, $0 \leqq p \leqq 1$.

Then:

a linear subspace of a space in $\mathscr{A}$ is, with the induced topology, a space on $\mathscr{A}$, providing its topology is not indiscrete;

the quotient of a space in $\mathscr{A}$ by a nondense linear subspace is, with the quotient topology, also a space in $\mathscr{A}$;

the completion of a $T_{1}$ space in $\mathscr{A}$ is also a space in $\mathscr{A}$.

Products. The product of any family of $r$-convex spaces is, with the product topology, $r$-convex.

The product of any family $\left\{X_{i}\right\}$ of upper bound spaces of type $p_{i}$ is, with the product topology, an upper bound space of type inf $\left\{p_{i}\right\}$.

The product of any finite family $\left\{X_{v}\right\}$ of locally bounded spaces of type $p_{v}$ is, with the product topology, a locally bounded space of type $\min \left\{p_{v}\right\}$.

The product of an infinite family of linear topological spaces can never be locally bounded.

Two examples. The space $l^{1} \times l^{1 / 2} \times l^{1 / 3} \times \cdots$ is a complete metric upper bound space of type 0 , i.e., an upper bound space but not $r$-convex for any $r>0$.

We suppose that $X$ is a complete metric locally bounded space of type 1 but not locally convex. (See the remarks following Definition 3.) Then the product of $X$ with itself a countable infinity of times is a complete metric upper bound space of type 1 but is neither locally bounded nor locally convex.

Boundedness. If $B$ is a bounded subset of a linear topological space $(X, u)$ and $f$ is any $u$-continuous invariant pseudometric on $X$ then $f(B)$ is a bounded subset of $R^{+}$. If $(X, u)$ is locally convex the reverse implication is true, i.e., if $B$ is a subset of $X$ and $f(B)$ is bounded for each $u$-continuous invariant pseudometric on $X$ then $B$ is bounded in $(X, u)$. This characterization of boundedness in terms of invariant pseudometrics turns out to be a very useful analytic device. We now show that this property of locally convex spaces does, in fact, extend to upper bound spaces.

THEOREM 6. If $(X, u)$ is an upper bound space and $B$ is a subset of $X$ such that $f(B)$ is bounded for each u-continuous invariant pseudometric $f$ on $X$ then $B$ is bounded in $(X, u)$. 
Proof. If $B$ were unbounded then we could choose a $u$-continuous $k$-pseudometric $f$ on $X$ such that $B$ was not absorbed by $\{x: f(x)<1\}$. For arbitrarily small nonzero $\lambda \in F$ there would exist $x \in B$ with $f(\lambda x) \geqq 1$. If $f(\lambda x) \geqq 1$ then $f(x) \geqq \lambda^{-k}$, and thus $f(B)$ would be unbounded.

The space $M$. We write $M$ for the set of (classes of) measurable functions on $[0,1]$, topologized by the invariant pseudometric

$$
d(x)=\int_{0}^{1} \frac{|x(t)|}{1+|x(t)|} d t .
$$

$M$ is a complete metric linear topological space. Nevertheless:

THEOREM 7. (a) Every continuous invariant pseudometric on $M$ maps $M$ into a bounded set.

(b) $M$ is not an upper bound space.

Proof. If $f$ is a continuous invariant pseudometric on $M$ then there exists $\delta \in R^{+}, \delta>0$, such that $d(x)<\delta$ implies that $f(x)<1$. Certainly $f(x)<1$ whenever $x$ has support of measure $<\delta$. Any element of $M$ can be expressed as the sum of $n$ elements with support of measure $<\delta, n=[1 / \delta]+1$, and thus, from the subadditivity of $f, f(x)<n$ for all $x \in M$. This proves the truth of (a).

(b) is immediate from (a), Theorem 6 and the observation that $M$ is not a bounded subset of itself.

Further question. Is there a linear topological space $(X, u)$, not an upper bound space, for which the property in Theorem 6 is true, or is this property in fact a characterization of upper bound spaces?

I would like to thank Professor V. Klee for reading through the manuscript of this paper and making a number of very helpful suggestions.

\section{REFERENCES}

1. A. E. Taylor, Introduction to functional analysis, Wiley, New York, 1958.

2. N. Bourbaki, Topologie générale, Actualités Sci. Ind., Hermann, Paris, 1948; Chapter 9.

3. D. G. Bourgin, Linear topological spaces, Amer. J. Math. 65 (1943), 637-659.

4. M. Landsberg, Lineare topologische Räume, die nicht lokalconvex sind, Math. Z. 65(1956), 104-112.

5. Robert Trull Ives, Semiconvexity and locally bounded spaces, $\mathrm{Ph}$. D. Thesis, Univ. of Washington, Seattle, Wash., 1957.

UNIVERSITY OF BRITISH COLUMBIA, Vancouver, B. C., Canada

Peterhouse,

CAmbridge, England 